\title{
¿UNA NUEVA SÍNTESIS? la agenda social de la izquierda uruguaya a dos años de gobierno
}

\author{
Carmen Midaglia* \\ Florencia Antia $a^{* *}$
}

\section{INTRODUCCIÓN}

Es común escuchar que las izquierdas, en particular en América Latina, han propuesto e implementado revisiones importantes de los sistemas de bienestar, como respuesta a los profundos cambios que esta área experimentó luego de la época de dominio de los modelos neoliberales. El estudio de algunos casos concretos permite discutir esa presunción general y plantear afirmaciones menos contundentes. Estas aseveraciones no niegan la ausencia de cambios, pero sugieren que la orientación se dirige más a un aumento de la intervención estatal en distintos campos sociales que a la reformulación de algunos modelos existentes.

En ese marco general, este artículo analiza el caso uruguayo y su evolución reciente. Se identifican las principales medidas sociales impulsadas en los primeros dos años de gobierno progresista, intentando interpretar su significado

* Professor-Doutor do Instituto de Ciência Política, Faculdade de Ciências Sociais, UdelaR, Uruguay. Constituyente 1502, Montevideo, Uruguay.midaglia@adinet.com.uy

* * Professor do Instituto de Ciência Política, Faculdade de Ciências Sociais, UdelaR, Uruguay. fantia@fcs.edu.uy político. Es así que se identifican las áreas o sectores públicos sujetos a modificaciones, la orientación predominante de las iniciativas promovidas, el papel que asume el Estado en torno a esos cambios y los efectos primarios que los mismos producen.

Los resultados sugieren la existencia de un empuje de estatismo moderado como el principal rasgo de los cambios en las áreas sociales, aún cuando muchas interrogantes permanecen planteadas sobre el recorrido futuro.

\section{EL NUEVO GOBIERNO DE IZQUIERDA Y LOS REFERENTES SOCIALESE INSTITUCIONALES DE BIENESTAR}

La izquierda uruguaya asume el gobierno en un contexto político-institucional y social específico que, de alguna manera, condiciona su manejo en materia políticas sociales. En primer lugar, como un aspecto favorable, interesa mencionar que esa fuerza política ganó las elecciones del 2004 en primera "vuelta electoral", obteniendo una mayoría parlamentaria $-51,7 \%$ de los votos váli- 
dos - que, si bien no le permite sancionar leyes especiales para las que se necesitan mayorías mas amplias que las actualmente dispone la bancada oficialista, igualmente le facilita la tramitación de una serie de medidas tendientes a modificar e innovar en materia social.

En segundo término, el gobierno tuvo que operar en un sistema de bienestar y protección que fue objeto de reformas y modificaciones en la década del 90, ya sea en la orientación de algunas de sus prestaciones, así como en los formatos de gestión. Sobre este tópico, importa señalar que la pauta de reforma que adoptó el país sobre el papel que asumiría el Estado y Mercado en la provisión social fue calificada de moderada (Castiglioni, 2005), respecto a la instrumentadas en otros países de la región. Independientemente de la moderación reformista en materia social, la resultante del proceso de revisión de la matriz clásica de bienestar dio lugar a un sistema "híbrido", en el que conviven servicios semi-privatizados con otros en los que se acentuó la intervención estatal, a los se anexan nuevas prestaciones de tipo focalizado, que poseen un formato mixto de implementación, es decir, de co-participación público-privado. A esta variedad de opciones de políticas sociales se agrega una significativa dispersión de los servicios al interior del entramado institucional público (Midaglia, 2006).

Por último, como otro aspecto de contexto a tener en cuenta, importa anotar que esta Administración se enfrentó a un escenario nacional pautado por las consecuencias sociales derivadas de la crisis económica del 2002 que agudizaron las situaciones de pobreza y exclusión que comenzaron a configurarse a partir de 1994.

Con un marco de referencia como el enunciado, el gobierno progresista pareció conducirse en el campo socioeconómico en dos rutas diferenciales, a saber:

- intentando responder al crítico panorama social con propuestas que se identificaran o poseyeran algún "sello de izquierda", y simultáneamente, controlando los parámetros macroeconómicos vigentes, para mejorar el desempeño económico del país, de acuerdo a los criterios de desarrollo imperantes internacionalmente;

- buscando cumplir con las promesas electorales, ya sea atendiendo las demandas de los actores colectivos históricamente aliados a esta fuerza política - sindicatos -, así como los planteos de grupos y sectores sociales afectados o descontentos con las reformas sociales instrumentadas en las anteriores administraciones.

La instalación del llamado "Uruguay Social”, en el que supuestamente tendrían lugar los distintos segmentos sociales, fomentándose así la recreación moderna de una "sociedad integrada", fue una de las banderas que levantó esta fuerza política en el período electoral del 2004. Cabe, entonces, preguntarse si la izquierda cumplió o está cumpliendo con sus promesas de campaña, así como con sus compromisos históricos, y a la vez logró imprimir a sus opciones de políticas sociales alguna impronta que la diferencie de las adoptadas por gobiernos de distinto signo político.

\section{EL CONTEXTO SOCIAL: el aumento de las vulnerabilidades sociales}

De modo a enmarcar la acción que ha emprendido el actual gobierno en el campo de la protección, resulta imprescindible presentar la situación social que ha caracterizado al país en los últimos 20 años.

Si bien Uruguay ha sido ubicado históricamente entre los países menos desiguales y pobres del continente latinoamericano, en los primeros años del siglo XXI esta situación tiende a desvanecerse. La fotografía actual de esta sociedad sugiere un proceso creciente de empobrecimiento, fragmentación social y pérdida de equidad.

Desde la recuperación de la democracia, la evolución de la pobreza en Uruguay recorrió cuatro etapas (De Armas, 2005; Amarante, et al., 2005). En una primera etapa (1985-1994), la incidencia de la pobreza descendió pronunciadamente (del 46,2 al 15,3\%); en una segunda etapa (1995-1999), la tendencia se revirtió y la proporción de hogares 
pobres comenzó a aumentar levemente; mientras que, en la tercera etapa (2000-2004), los niveles de pobreza se incrementaron significativamente, alcanzando a poco más de un tercio de la población (31,9\% en 2004). Finalmente, en la cuarta etapa, que se inicia en 2005, se advierte una caída de la incidencia de la pobreza, que en 2006 se situó en un 25,2\% de la población. A pesar de la evolución favorable del último bienio, la pobreza se ubica todavía en niveles superiores al período previo a la crisis del año 2002. ${ }^{1}$

Gráfico 1 - Evolución de la pobreza (Líneas de pobreza 1996 y 2002) (1986-2006)

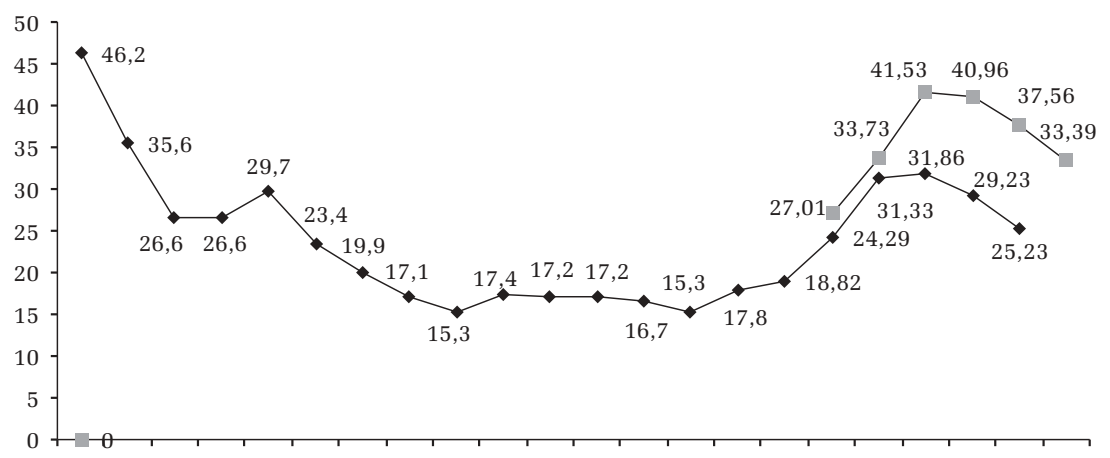

$\begin{array}{lllllllllllllllllllll}86 & 87 & 88 & 89 & 90 & 91 & 92 & 93 & 94 & 95 & 96 & 97 & 98 & 99 & 00 & 01 & 02 & 03 & 04 & 05 & 06\end{array}$

$\multimap$ Pobreza LP02 —- Pobreza LP96

Fuente: INE, 2002. Amarante y Vigorito, 2007.

Gráfico 2 - Incidencia de la pobreza por tramos de edad (LP 1996) (2001-2006)

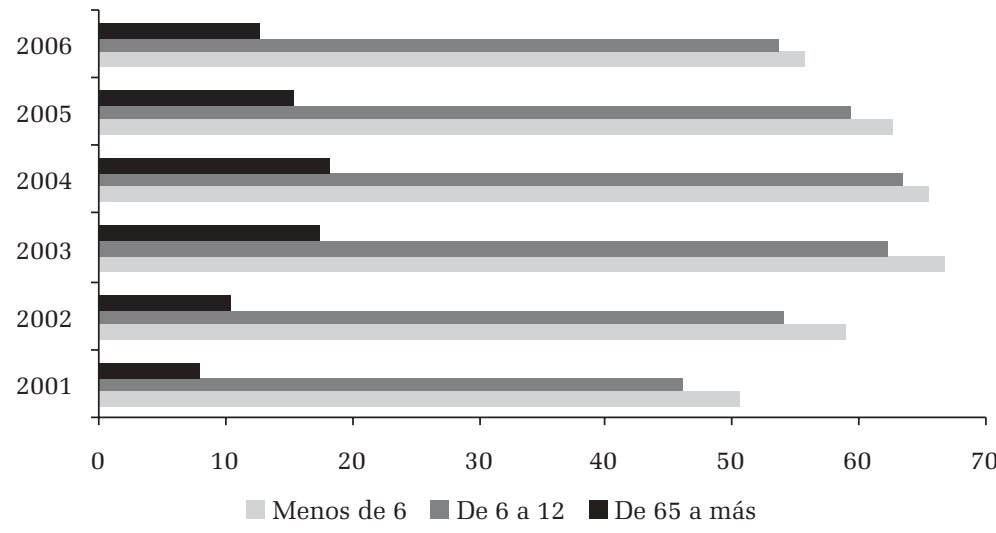

Fuente: Elaboración propia en base a Amarante y Vigorito (2007).

${ }^{1}$ Los datos de pobreza aquí planteados se basan en la medición de la pobreza por el método del ingreso, que consiste en comparar el ingreso per cápita de cada hogar con un umbral monetario. El INE produce información sobre pobreza en función de dos líneas, una desarrollada
Un aspecto a destacar es la particular incidencia de la pobreza entre los niños y jóvenes, con relación a otros grupos de edad. En tal sentido, en 2001 la pobreza alcanzaba al 50,53\% de los niños menores de 6 años, mientras que llegaba al 8,02\% de los mayores de 65 años. Los niveles de pobreza en la infancia aumentaron en los últimos años, y en 2006 escalan a un 55,74\% de los niños menores de 6 años. La disparidad generacional señalada tendió a reducirse a partir de la última crisis económica, como consecuencia de la pérdida del valor real de las pasividades (Amarante; Vigorito, 2007).

Una trayectoria similar a la planteada para la pobreza se constató en relación a las situaciones de indigencia. El porcentaje de personas en dicha situación aumentó significativamente durante la crisis económica del 2002, pasando de ubicarse en $2,03 \%$ en 2001 a $5,99 \%$ en 2004. Sin embargo, en el año 2006 ese porcentaje descendió significativamente, ubicándose en un 2,69\% de la población (Amarante; Vigorito, 2007). ${ }^{2}$

No sólo la pobreza fue en aumento en el país sino también los grados de desigualdad social. Desde la segunda mitad de la década de los 90 se constata una leve tendencia concentradora en la distribución del ingreso, que se tendió a agudizar en el período de la crisis económica. Como se

en 1996 (por el Taller Regional sobre Medición de la Línea de la Pobreza 1996) y otra en 2002. En este marco, hemos optado por incluir los datos disponibles correspondientes a la Línea de Pobreza elaborada por el INE en 1996 y en 2002.

${ }^{2}$ Aquí se toma la línea de indigencia 1996. 
advierte en el gráfico, el índice de Gini pasó de 0,412 en 1991 a 0,450 en 2002, para finalmente situarse en 0,447 en $2006 .^{3}$

mantuvieron con escasas modificaciones en los primeros dos años de ejercicio de este gobierno, pese a incrementarse el gasto público destinado a Gráfico 3 - Evolución de la distribución del ingreso en Uruguay (1991-2006)

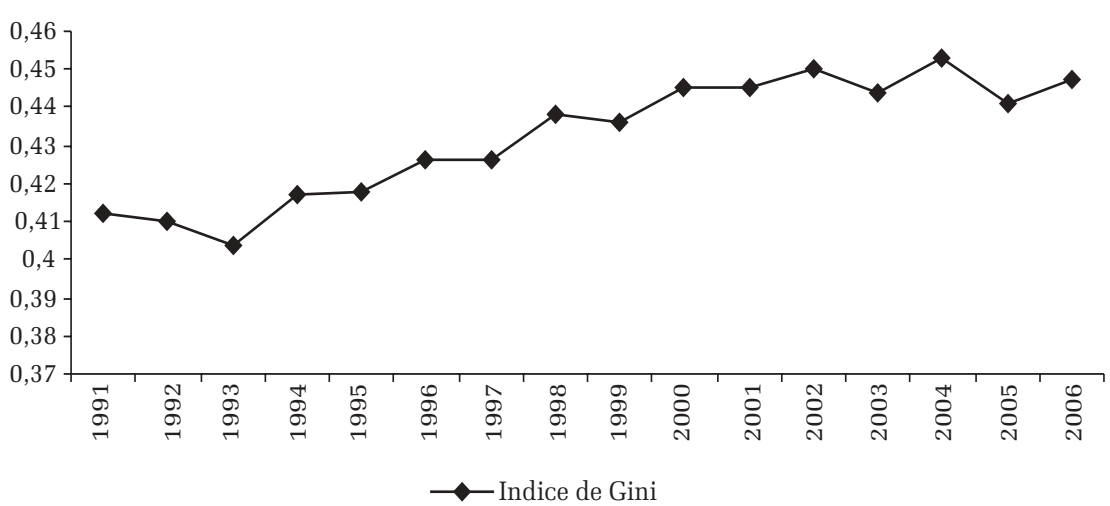

Fuente: IDH, 2005 (para los años 1996-2004), Amarante y Vigorito, 2007 (para los años 2005-2006).

las mismas. Esta situación parece revertirse en particular para el sector salud, en la medida que se anunció a mediados del año 2007 una ambiciosa reforma sanitaria, de la que se ejecutará en este mandato una primera fase.

Por otra parte, los distintos servicios semiprivatizados-seguridad social - y aquellos que han tercerizado su instrumentación, generalmente asociados a nuevas demandas sociales infancia, juventud y género-se conservaron sin may-

\section{EL URUGUAY SOCIAL: ¿estrategia discusiva o promesa en fase de cumplimiento?}

Es prematuro y aventurado evaluar el conjunto de propuestas sociales que instrumentará el gobierno progresista hasta el fin de su mandato (2009). No obstante, las medidas ya puestas en práctica, a las que agregan otras recientemente planteadas, permiten identificar un paquete de iniciativas sociales que tienden a diferenciarse de las promovidas en períodos anteriores.

Resulta pertinente afirmar que la izquierda política uruguaya centró su acción en el campo público social hasta el presente en tres arenas específicas: la laboral-regulaciones; la vinculada a pobreza-planes focales; y la asociada con la distribución o redistribución de la riqueza que trasciende el campo típicamente social-reforma tributaria.

Las áreas sociales vinculadas a la inversión en capital humano - educación y salud - se

\footnotetext{
${ }^{3}$ El Índice de Gini ofrece un resumen de la información de la distribución de los ingresos de toda la población, expresando cuánto se aleja la distribución real del ingreso respecto de la distribución perfectamente igualitaria. El coeficiente de Gini varía entre 0 y 1 , siendo que los mayores valores indican una distribución de ingresos más desigual.
} ores cambios, pese a que, en su proceso de reformulación, se desataron amplias críticas y oposiciones de parte de gremios, sindicatos, entre otros actores colectivos, a los que agregaba la propia izquierda política.

\section{Las arenas públicas privilegiadas por la izquierda}

Como era de esperar, el ámbito laboral fue uno de los campos preferidos de intervención del gobierno. La tradición de la izquierda con las organizaciones de trabajadores y la defensa de los derechos laborales transformaron a esta fuerza política en portadora de los intereses de ese sector social o de "clase". Históricamente los partidos de izquierda y los dirigentes sindicales guardaban un estrecho vínculo, que hasta avanzada la década del 70 no se tradujo necesariamente en apoyo electoral a esos partidos clasistas. Esta disociación entre voto y afiliación o simpatía sindical dio lugar a lo que designó como el Uruguay de la "doble escena" (Solari, 1967), en la medida que, en los períodos inter-electorales, los gremialistas acompañaban en sus acciones a los dirigentes sindicales y, en los 
períodos electorales, seguían a los representantes de otros partidos (los partidos tradicionales). Este camino paralelo comenzó a entroncarse a partir de la apertura democrática, cuando los partidos izquierda, reunidos en la coalición Frente Amplio, amplían sus bases electorales, no sólo incorporando a una amplia gama de trabajadores, sino también a sectores sociales pobres de la población, al menos en la capital del país (Moreira, 2005).

\section{La regulación laboral}

En este marco de vinculación entre la izquierda y los sindicatos no resulta sorprendente que una de las primeras medidas sociales que promulgó este gobierno se inscribiera en la esfera laboral. Es así que se reinstalaron los Consejos de Salarios, que suponen nada más ni nada menos que la activación de los mecanismos de negociación colectiva para dirimir los típicos conflictos distributivos entre el capital y trabajo. La aplicación de esta política es altamente significativa luego de un extenso período de desregulación laboral en el que predominó una drástica disminución de las negociaciones salariales, y, cuando éstas existieron, se enmarcaron sólo en algunas ramas productivas, fundamentalmente aquellas que presentaban una correlación de fuerza favorable a los trabajadores sector financiero. Esa fase de desregulación laboral fue acompañada del surgimiento de programas orientados a la recalificación de la fuerza de trabajo desocupada, bajo el supuesto que la simple capacitación de los trabajadores tendría como resultado una reincorporación rápida o inmediata al mercado de trabajo. Esta relación entre capacitación e inserción laboral no logró constituirse en una sólida y contrastable tendencia (Midaglia, et al., 2006).

La puesta en marcha de los Consejos de Salarios produjo un mejoramiento de las pautas salariales de diversos sectores de trabajadores, a la vez que fortaleció a las organizaciones de representación de los intereses presentes en esas esferas, sindicatos y cámaras empresariales. Una de las novedades en la aplicación de estos meca- nismos radicó en que, por primera vez en la historia, se incorporan a los asalariados rurales.

Interesa señalar que, desde la consagración de estas instancias de negociación, los sindicatos pertenecientes a la Central Única de Trabajadores - PIT-CNT - no sólo aumentaron su nómina de afiliados en el entorno de los 100.000 trabajadores, sino que también se registró la creación de 400 nuevas organizaciones sindicales (Senatore, 2007). En materia de resultados salariales, se advierte una recuperación del salario real público y privado. En tal sentido, en diciembre de 2006, el Índice medio de salarios fue un $11,5 \%$ superior que en junio de 2004 (Notaro, 2007).

Si bien los Consejos de Salarios cubren una magnitud importante de trabajadores $-440.000 \mathrm{del}$ sector privado, 150.000 del público, y 80.000 asalariados rurales - aún resta incorporar a las trabajadoras del servicio doméstico, los trabajadores por cuenta propia y los trabajadores rurales no asalariados (Notaro, 2007). Más allá de estos problemas de cobertura, importa mencionar el dinamismo que han tenido los Consejos de Salarios, en la medida que se registra una significativa productividad en torno a la celebración de acuerdos laborales. En el sector privado, el 96\% de los casos arribaron a acuerdos de esa naturaleza (Ministerio de Trabajo y Seguridad Social, 2006).

\section{Pobreza y vulnerabilidad}

Otras de las áreas sociales priorizadas por esta Administración fue la vinculada a las situaciones de pobreza y vulnerabilidad social. Si bien el desfavorable contexto social en el que la izquierda asumió el gobierno jugó un papel clave en la definición de respuestas en torno a esas situaciones, interesa señalar que recién en los últimos veinte años la izquierda jerarquizó y problematizó la temática de la pobreza.

Cabe recordar que Uruguay contó con una matriz temprana de bienestar y protección que se remonta a las primeras décadas del siglo XX y se extendió, aunque con variaciones, hasta la fase de 
reapertura y consolidación democrática de mediados de los años 80' y 90'. (Castelleno, 1996; Filgueira, 1998; Moreira, 2003). Ese sistema se había "edificado" en torno a un mercado dinámico de empleo que, en caso de fallar, recurría a la acción política para ampliar el margen de protección. Esta operativa se hacía a través de modalidades particularistas - clientelares - que se inscribían en una estructura plural de partidos y fracciones políticas, a la que se agregaba una serie de actores sociales. En un entorno político distributivo, caracterizado por un equilibrio relativo de fuerzas, calificado por algunos analistas como un escenario de balances y contrabalances (Filgueira, 1998), resultaba imposible la emergencia y tramitación de un proyecto socioeconómico hegemónico.

No había lugar, en ese pasado reciente, para el "desamparo total" de los ciudadanos. Con la primacía en la región de los enfoques desarrollistas, la pobreza se consideraba relativamente transitoria, producto de procesos migratorios campo-ciudad. Su superación pasaba a depender de una suerte de combinación de un mercado de trabajo activo con la acción permanente de un Estado proveedor de bienes sociales de relativa calidad.

Por esta razón, la atención de los sectores de izquierda se centró tradicionalmente en las relaciones capital-trabajo, en la medida que estas influían en las condiciones de vida de la mayoría de la población.

Ahora bien, sin abandonar sus clásicos temas, las izquierdas en general, incluidas las latinoamericanas, comenzaron a preocuparse por identificar alternativas tendientes a mejorar las persistentes situaciones de vulnerabilidad y exclusión social que se extendían y/o agudizaban en la región, a partir de la redefinición de los parámetros internacionales de desarrollo. La búsqueda de mecanismos que aliviaran las complejas problemáticas sociales comenzó a formar parte de la nueva agenda política regional, en la medida que la experiencia internacional acumulada evidenciaba que, si bien el crecimiento económico contribuía a mejorar dichas situaciones, se requería a la vez de intervenciones públicas per- manentes que abordaran la multidimensionalidad de las situaciones de pobreza y, simultáneamente, incidieran en la estructura de inequidad imperante (CEPAL, 2006).

El actual gobierno suscribió este tipo de preocupaciones sociopolíticas, y su traducción práctica supuso la promoción de tres medidas de distinta naturaleza y proyección, pero altamente significativas para el sistema de protección nacional. Dos de ellas refieren a programas dirigidos a cubrir las necesidades básicas o específicas de grupos en situación de exclusión y/o vulnerabilidad social: Plan de Atención a la Emergencia Nacional - PANES -, de naturaleza coyuntural, y el Plan de Equidad, que se concibió como una propuesta estable.

La tercera medida, en cambio, fue de carácter estrictamente institucional, referida a la creación del Ministerio de Desarrollo Social, encargado de instrumentar y monitorear los planes mencionados, a la vez que coordinar el conjunto de prestaciones sociales que brinda el Estado.

El Plan de Emergencia se proyectó como una intervención pública transitoria, diseñada para operar en los dos primeros años de gobierno. La misma se estructuró en base a siete componentes que garantizaban una intervención integral del Plan. Entre los más destacados figuran: el ingreso ciudadano; el apoyo alimentario y el alojamiento de personas en situación de calle. Este programa focalizó su acción en los sectores sociales calificados de indigentes, es decir, aquellos grupos que presentan serias dificultades para asegurar su reproducción física. Más allá que esta iniciativa presentó algunos problemas de instrumentación en las fases iniciales, en el momento actual y a escasos meses de su finalización, es adecuado calificarla de exitosa, en la medida que alcanzó sus objetivos y la meta de cobertura estipulada (Midaglia, 2006). ${ }^{4}$

El Plan de Equidad, por su parte, se diseñó como una propuesta de largo plazo, que pretende

\footnotetext{
${ }^{4}$ El Plan de Emergencia atiende a 76.991 hogares que se traducen en 337.240 personas destinatarias de esta iniciativa (MIDES, Dirección de Evaluación y Monitoreo, 2006).
} 
incidir en la estructura de desigualdades vigente en el país, ya sea de tipo socioeconómico, de género, de etnia, regionales, entre otras. No obstante, en el corto plazo, se enfoque en las situaciones de carencia socioeconómica, pero, en esta oportunidad, intenta cubrir a sectores más amplios de pobreza que los incluidos en el Plan Emergencia. En esta primera etapa, pretende instalar una "malla" de protección social, reutilizando instrumentos clásicos de amparo - Asignaciones Familiares que se orientan a corregir fundamentalmente los desbalances sociales intergeneracionales en favor de la infancia y juventud, ya que esos grupos son los más vulnerables. También se incluyen intervenciones complementarias, dirigidas en particular a los jefes de hogares pobres. Adicionalmente, se prevé la atención a los adultos mayores (Midaglia, 2007).

Si bien estas propuestas son relativamente novedosas para el sistema de bienestar y protección uruguayo, caracterizado por la orientación universal de la mayoría de sus servicios, importa mencionar que el país disponía de ciertos antecedentes al respecto. Por una parte, se había promovido, en los años 90, una ampliación y reformulación de un conjunto de intervenciones dirigidas a la infancia y juventud carenciada. Por otro lado, las Administraciones anteriores suscribieron préstamos internacionales para el diseño de programas de naturaleza similar pero menos ambiciosos - por ejemplo, el programa Infamilia.

Vale la pena señalar que esos modernos Planes, específicamente los que incorporan entre sus prestaciones transferencias de ingresos condicionadas, se han convertido en una estrategia de acción generalizada en el continente, y por ende no parecen ser un instrumento propio de los gobiernos de izquierda latinoamericanos. Tal vez la impronta ideológica de las Administraciones de izquierda, incluyendo la uruguaya, radique en la consideración explícita que estos programas ofrecen oportunidades de generar articulaciones estables con el resto los servicios públicos sociales, de manera de construir un sistema permanente de protección o asistencia social.
La puesta en marcha de estos Planes se enmarcó en el flamante Ministerio de Desarrollo Social. La instalación de esta entidad procura, en alguna medida, reorganizar la esfera de la asistencia pública, no sólo instrumentando y coordinando la ejecución de los mencionados planes, sino que también incorporando, en su órbita, una serie de institutos sociales dispersos en el entramado público - INAU, INAMU, INJU ${ }^{5}$ - que dan cuenta de nuevas demandas sociales.

La creación de ministerios sociales especializados en el tratamiento de situaciones vinculadas con la pobreza y con problemáticas específicas es otra de las medidas que también se extendieron en Latinoamérica. Para aquellos países que contaban con sistemas maduros de bienestar y que los procesos de reforma socioeconómica no los había desmontado radicalmente, el establecimiento de esas entidades resulta institucionalmente significativo.

En Uruguay, la reorganización de los servicios sociales se vislumbra como necesaria e imprescindible, no sólo en términos de la mejora de la eficiencia del gasto público, sino que para introducir coherencia y complementariedad en el sistema de políticas sociales. A pesar de la importancia instrumental de esa tarea, su puesta en práctica por parte de estos ministerios es políticamente compleja, ya que supone, en última instancia, redistribuciones de funciones y poder al interior de la esfera pública. Algunos estudios nacionales constatan que una proporción importante de organizaciones estatales ofrecen, sin mayor coordinación, servicios sociales de similar característica, favoreciendo la superposición de prestaciones y generando a la vez múltiples "bocas de entrada" para adquirir un conjunto de bienes (MIDES-UdelaR, 2007).

${ }^{5}$ INAU: Instituto del Niño y del Adolescente del Uruguay; INAMU: Instituto Nacional de las Mujeres; INJU: Instituto Nacional de la Juventud. 


\section{La reforma tributaria}

Una de las modificaciones estructurales de mayor relevancia que lleva a cabo el gobierno de izquierda es la reforma tributaria. Si bien no es una medida estrictamente social, resulta un instrumento privilegiado de distribución o redistribución de la riqueza.

La reforma tributaria formó parte de la agenda programático-electoral del Frente Amplio por largos años, y, específicamente, el Impuesto a la Renta de las Personas Físicas (IRPF) aparece como una de las propuestas distintivas de esta fuerza política.

El proyecto de reforma fue elaborado por un equipo que trabajó en el marco del Ministerio de Economía y Finanzas. Luego de un largo trámite parlamentario - que implicó una compleja negociación al interior del propio Frente Amplio la reforma tributaria fue aprobada y comenzó a implementarse recientemente, en julio de 2007. Los objetivos que persigue son: i) generar mayor equidad del sistema impositivo, relacionando la carga tributaria con la capacidad de contribución de los distintos sectores sociales y económicos; ii) incrementar la eficiencia del sistema; iii) estimular la inversión productiva y el empleo; y iv) satisfacer las necesidades financieras del Estado (Ley de reforma del sistema tributario. Exposición de motivos, 2006). En tanto, la propuesta se apoya en cuatro pilares básicos: i) la simplificación de la estructura impositiva; ii) la racionalización, en procura de una mayor consistencia entre las características de los diversos tributos; iii) la introducción del IRPF, como instrumento capaz de mejorar la equidad del sistema; y iv) un enfoque dinámico de la responsabilidad fiscal, que asegure el funcionamiento del Estado, y que, a la vez, permita reducir la carga impositiva a medida que la recaudación lo permita (Ley de reforma del sistema tributario. Exposición de motivos, 2006). ${ }^{6}$

\footnotetext{
${ }^{6}$ No se realizará aquí una descripción detallada de todos los componentes del nuevo sistema tributario, sino que se considerarán aquellas modificaciones que puedan tener mayores impactos distributivos.
}

El impuesto a la renta es una variante del denominado sistema de imposición "dual”, cuya principal característica es el tratamiento diferencial a las rentas del trabajo, respecto de las rentas del capital (las primeras se someten a una tasa progresiva, mientras que la segunda a una tasa fija). El mínimo no imponible del IRPF deja fuera del impuesto un elevado porcentaje de la población, que llega a casi el $60 \%$. Este aspecto se diferencia del régimen anterior, en que sólo el 30\% de los asalariados estaban exonerados del Impuesto a las Retribuciones Personales (IRP) (Perazzo; Rodríguez, 2006).

Por otra parte, se reduce la imposición al consumo, con lo que se procura disminuir el peso de los impuestos indirectos en la estructura tributaria global - que, en el régimen anterior, alcanzaba al 70\% de la recaudación total (Perazzo y Rodríguez, 2006) -, en el entendido que estos tributos tienden a ser regresivos. ${ }^{7}$ Entre otras innovaciones relevantes, cabe mencionar las modificaciones al régimen de Monotributo, en procura de que éste se convierta en una herramienta de inclusión social y formalización del trabajo. En tal sentido, se persigue la incorporación de trabajadores que desarrollan actividades empresariales de reducida dimensión económica, quienes pagarán un único tributo (en sustitución de los demás impuestos nacionales y con un monto relativamente bajo) y accederán a los beneficios de la Seguridad Social.

Si bien resulta arriesgado adelantar los impactos distributivos de la reforma tributaria, las estimaciones oficiales señalan que los cambios en la imposición general al consumo y a la renta personal supondrían un aumento del ingreso disponible de los hogares que se ubican entre los deciles 1 y 8 , mientras que los hogares correspondientes a los deciles más ricos (9 y 10) experimentarían una disminución del ingreso disponible a raíz de una mayor carga tributaria. En líneas generales, diver-

${ }^{7}$ No obstante, se agregan a la base imponible del IVA otros bienes y servicios que, en el régimen anterior, se encontraban exonerados de este impuesto. 
sos estudios indican que el nuevo sistema tendría efectos positivos en términos de equidad. No obstante, habrá de esperar las mediciones de los resultados de la reforma tributaria una vez implementada, de modo de conocer los impactos sociales del nuevo sistema.

Más allá de estas expectativas, lo cierto es que la reforma ha despertado la crítica de diversos sectores políticos y sociales, especialmente de los partidos tradicionales y de algunos sindicatos históricamente próximos al Frente Amplio (como es, por ejemplo, el gremio de empleados bancarios).

\section{El rol de la izquierda en la inversión en capi- tal humano}

\section{Salud}

A poco más de dos años de ejercicio de gobierno, se ha comenzado a instrumentar una ambiciosa reforma en el ámbito de la salud, orientada a crear un Sistema Nacional Integrado de Salud (SNIS). ${ }^{8}$ El principal objetivo del nuevo sistema es asegurar el acceso universal a los servicios de salud, guiado por principios de equidad, calidad y descentralización (Exposición de motivos. Ley de creación del SNIS).

Esta propuesta emerge ante un panorama del sistema de salud que se muestra especialmente crítico. Desde la recuperación de la democracia, se han sucedido una serie de proyectos de reforma de ese sistema, que resultaron en su mayor parte fallidos. Los bloqueos políticos y corporativos obstaculizaron la tramitación de los mismos (Fernández, et al., 1999). De ahí que el sistema fuera sometido a un proceso de reestructuración pasivo (Moreira; Setaro, 2002) y muestre, en la actualidad, un estado de deterioro en términos de la calidad de sus servicios y de la equidad (Pereira et al., 2005).

${ }^{8}$ En este marco, se han elaborado tres proyectos de ley: la ley de creación del Fondo Nacional de Salud (FONASA), ya aprobada por el Parlamento, el proyecto de creación de un SNIS y el proyecto de ley de descentralización de ASSE, ambos a estudio del Parlamento.
Vale la pena plantear brevemente la forma en que se ha estructurado el sistema de salud en las últimas décadas, para luego analizar la orientación de la propuesta de reforma en trámite. El antiguo sistema presentaba un carácter mixto, donde el sector público se orientaba a la atención de la población de más bajos recursos (y, en términos generales, más joven), y el sector privado a los de recursos medios y altos. En este marco, los trabajadores formales gozaban de un seguro de salud obligatorio administrado por la Dirección de Seguros de Salud del Estado (DISSE) y prestado por las instituciones de asistencia médica colectiva (IAMC). Según los datos de la Encuesta de Hogares Ampliada referidos a 2006, el 50,8\% de la población se atiende en las instituciones públicas, un 45,7\% en el sistema privado (mayoritariamente en las IAMC), y, finalmente, un 2,5\% de la población declara no tener cobertura (Trylesinski, 2007, p. 7). Cabe señalar que las mutualistas, que brindan cobertura a casi la mitad de la población, han atravesado, en los últimos años, por una profunda crisis financiera, al tiempo que el sector público presenta también serias deficiencias.

Ante un panorama sectorial tan complejo, la administración frenteamplista creó un equipo en el Ministerio de Salud Pública encargado de diseñar la reforma. Recientemente se comenzaron a implementar los primeros pasos hacia un nuevo sistema integrado de salud.

De acuerdo al proyecto de ley, las innovaciones se plantearán en tres planos: i) en el modelo de atención de salud, a través de la jerarquización del primer nivel de atención; ii) en la forma de gestión, mediante la articulación de servicios públicos y privados, con un órgano central de gobierno, la Junta Nacional de Salud; y, finalmente, iii) en el sistema de gasto y financiamiento del sector, a través de la creación de un fondo único salud - FONASA - administrado centralmente. Dicho fondo se financiará en base a las contribuciones del Estado y de las empresas privadas en forma proporcional al personal a su cargo. Así como también aportarán - de acuerdo a sus ingresos - los trabajadores públicos y priva- 
dos, los pasivos y quienes perciban rentas. Finalmente, se prevé el aporte fiscal a través de partidas presupuestales y extra-presupuestales que se le asignen.

Los usuarios del sistema tienen derecho a su propia cobertura de salud, la de sus hijos menores de 18 años y, en el futuro, de sus cónyuges. Otra de las novedades de la reforma es que incorpora a los trabajadores públicos (30.000) al sistema de salud.

Las prestaciones de salud estarán a cargo de las IMAC, las Instituciones de Asistencia Médica Privada Particular sin fines de lucro, y de los seguros integrales que estén funcionando regularmente, a lo cuales se le agregan los servicios de salud a cargo del Estado - ASSE -, que pasarán a competir con los prestadores privados.

Una de las innovaciones más relevantes que introduce la reforma es que el FONASA pagará una "cuota salud" a las instituciones prestadoras integradas al sistema, de acuerdo con los servicios que brinden. Las cuotas salud variarán en función de los riesgos de los diferentes sectores de la población, los que se determinarán por el sexo y la edad de los usuarios. Con ello se busca que las instituciones prestadoras atiendan a personas de cualquier edad, evitando así estímulos a la "selección adversa", es decir, que se prioricen, como en la actualidad, a los grupos que experimentan menores problemas sanitarios (Olesker, 2007).

La iniciativa tiende a jerarquizar a las instituciones de salud pública y, con ello, cabe suponer que mejorará la asistencia de los segmentos de población que se atienden en esa esfera. Más allá de los potenciales impactos positivos de carácter social y asistencial de la reforma de salud proyectada, la misma aún tiene que sortear importantes vetos políticos, así como la oposición de grupos o "seudocorporaciones" que se beneficiaban, o al menos no se perjudicaban, con el sistema anterior.

\section{Educación}

La educación pública no ha sido objeto de revisiones globales por la actual Administración.
Los niveles primario y medio formaron parte del proceso reformista de la década de los 90;, pero, a diferencia de la orientación regional dominante en este y otros sectores, su formato de ajuste supuso una mayor participación del Estado y una orientación universal. En tal sentido, la reforma de 1995 promovió modificaciones generales a la currícula y en la carga horaria de la enseñanza media, expandió la educación inicial de 4 y 5 años, introdujo programas focales para aquellos contextos socioeconómicos críticos (a través de las escuelas de tiempo completo) y extendió la formación de profesores en el interior del país (Lanzaro, 2004).

La reforma se basó en un amplio diagnóstico respecto los problemas de la educación, desarrollado desde la oficina de la CEPAL en Montevideo. Si bien su implementación despertó fuertes críticas y suscitó la oposición de los gremios docentes y estudiantiles, los principales lineamientos de la reforma no fueron modificados radicalmente por las sucesivas administraciones, hasta el presente.

Actualmente, el sistema educativo uruguayo presenta algunos déficits significativos. Uno de los principales refiere a la insuficiente cobertura en la educación media, que alcanza al 85,1\% de los adolescentes entre 12 y 17 años en 2006. Junto a ello, en este nivel se identifica un problema de rezago y de deserción, especialmente asociados a la pobreza y al género (Katzman; Rodríguez, 2007, p.31). Por otra parte, si bien el ciclo inicial ha expandido significativamente su cobertura en los niños de 4 y 5 años, es posible identificar un déficit de cobertura en las primeras edades, que se manifiesta en que sólo el 48\% de los niños de 3 años asistían en 2006 al sistema educativo (Katzman; Rodríguez, 2007). Aparte de los problemas mencionados, otros estudios han identificado resultados insatisfactorios de los aprendizajes en educación primaria y media, así como desigualdades en los logros de los alumnos de distintos contextos socioeconómicos (ANEP, 2002).

Ante este panorama, la administración frenteamplista no ha introducido, hasta ahora, modificaciones significativas al sistema, y al pare- 
cer no se disponen de plazos políticos para iniciar una acción transformadora del mismo en esta gestión de gobierno. ${ }^{9}$ Sin embargo, esto no significa que el gobierno le reste importancia a la educación, en la medida que se le asignó incrementos presupuestales, al tiempo que se mantiene la promesa electoral de alcanzar en el final del mandato el 4,5\% del PBI.

El comportamiento gubernamental, escasamente propositivo en este campo, se tendió a "corregir" con la promoción de una iniciativa particular: el llamado Debate Educativo llevado a cabo en el año 2006. En esta instancia de amplia participación, al menos de los gremios de la educación pública, surgieron una serie de propuestas que posteriormente se elevaron a las autoridades de la enseñanza y a representantes políticos, así como recomendaciones para la elaboración una nueva Ley de Educación. (Mancebo; Bentancur, 2007).

Es de suponer que el Debate Educativo se constituyó en un instrumento político que persiguió distintos objetivos. Uno de ellos estaría referido a la generación de un consenso que permitiera incorporar los ajustes necesarios para mejorar el funcionamiento y los resultados sociales de este sector. Otro de los objetivos buscados podría implicar una decisión política de postergar el tratamiento de este sector para un futuro inmediato, ya sea por la alta conflictividad que desencadenaría la incorporación de algunos cambios si se atiende la fortaleza y posición de los actores involucrados, así como por la necesidad de priorizar otras áreas de reconversión pública en este período de gobierno (Midaglia; Antía, 2007).

Por último, interesa mencionar un problema adicional en el campo de la educación, relativo a que la orientación estatista de la reforma, establecida en los noventa, no parece distanciarse de la posición ideológica que sustenta la coalición de izquierda en materia de políticas públicas.

${ }^{9}$ Entre las modificaciones, cabe destacar la reformulación de los planes de educación secundaria y los programas Maestros Comunitarios y Aulas Comunitarias, orientados a brindar apoyo pedagógico específico a niños y adolescentes con bajo rendimiento (en un acuerdo ANEPMIDES-INFAMILIA).

\section{LA PERMANENCIA DE LOS SERVICIOS SEMIPRIVATIZADOS}

El sistema de Seguridad Social, particularmente el de jubilaciones y pensiones, no parece encontrarse incluido en la agenda de revisiones del gobierno, pese a que el Frente Amplio fue muy crítico sobre el tipo de reforma de la que fue objeto el sector en 1995. A partir de la aprobación de la Ley 16.713, se habilitó el cambio de esquema de seguridad social, sustituyéndose el clásico sistema de monopolio estatal por otro de naturaleza mixta. Se estableció un régimen asentado en dos pilares diferenciales, pero que mantienen vinculación: uno de ellos, de solidaridad o reparto intergeneracional propio del sistema anterior, gestionado por un organismo público - el Banco de Previsión Social, BPS -, y otro, nuevo, de capitalización individual, administrado por agentes privados - Administradoras de Fondos de Ahorro - AFAP ${ }^{10}$ (Busquets, 2002; Castiglioni, 2005).

Seguramente la posición que asumió esta Administración, en torno a la reforma social que tuvo el sesgo más privatizador entre el repertorio de cambios instrumentados en los períodos anteriores, se explique por un conjunto diverso de factores, entre los que figuran: la ausencia de un acuerdo en el gobierno sobre la alternativa sustitutiva $^{11}$; la presencia de otros puntos de veto políticos de importancia - las administradoras de fondos de retiro, AFAPs; los sectores socioeconómicos beneficiados por el sistema vigente; los costos económicos-financieros que

10 Previo a la aprobación de esa reforma, en 1989 se introdujo, a través de un plebiscito, una enmienda constitucional que modificaba la pauta de ajuste de las pensiones y jubilaciones, la que posibilitó que, en diez años, se duplicara el valor real de estas prestaciones. Si bien esa modificación impactó positivamente en la situación de pobreza de más de un tercio de la población beneficiaria, simultáneamente generó un aumento del gasto público que se tradujo en una expansión del déficit fiscal. La preocupación política frente a esta situación se plasmó en la elaboración de un conjunto de propuestas de cambio del sistema que no tuvieron el apoyo suficiente para su tramitación.

11 Algunos sectores gubernamentales sostienen la necesidad de eliminar el pilar de capitalización individual, mientras otros rechazan esta posibilidad y proponen diversos ajustes menores. 
implicaría una nueva revisión del sistema y el "tiempo político disponible" para instrumentar modificaciones en el marco de una agenda de reformas muy cargada.

Sin embargo, el gobierno no eludió el tema, ya que, a través de la Comisión Sectorial de Seguridad Social promovió, la realización de un Diálogo Nacional sobre este tópico, intentando así crear un ámbito en el que técnicos-especialistas, actores sociales y representantes del gobierno discutan sobre los principales problemas que presenta el sistema y planteen propuestas tendientes a su mejora. (Busquets, 2007).

No hay lugar a duda que la estrategia política del Diálogo se presenta como un atajo que permite postergar la definición de una propuesta concreta de cambio, y simultáneamente fomenta la generación de un ambiente de intercambio de visiones, no exento de conflictos, en el que discutir y evaluar la viabilidad de diversas alternativas correctivas del sistema.

De cualquier manera, se estima la necesidad de introducir algunas modificaciones en el futuro inmediato, ya que los diagnósticos disponibles constatan una serie de problemas relevantes del sistema reformado de seguridad social. Se evidencia un alto riesgo de que un porcentaje muy significativo de los trabajadores cotizantes del BPS quede excluido del régimen general de jubilaciones en los próximos años, al no lograr alcanzar el requisito de 35 años de servicio al llegar a las edades usuales de retiro ${ }^{12}$ (Bucheli, et al., 2006). Esta situación no se presenta como propia del país, sino que es compartida por un conjunto amplio de sistemas privatizados de la región (CEPAL, 2006).

\section{UN MANEJO “RESPONSABLE” DEL GASTO PÚBLICO SOCIAL}

El conjunto de reformas y modificaciones sociales impulsadas por este gobierno supuso un incremento moderado del gasto público social.

Tradicionalmente, Uruguay ha presentado un elevado nivel de gasto público social, en comparación con los países de la región latinoamericana (CEPAL, 2006). En el Gráfico 4, se observa la evolución del gasto público social

Gráfico 4 - Evolución del gasto público social como porcentaje del PBI (1980-2005) (en porcentajes)

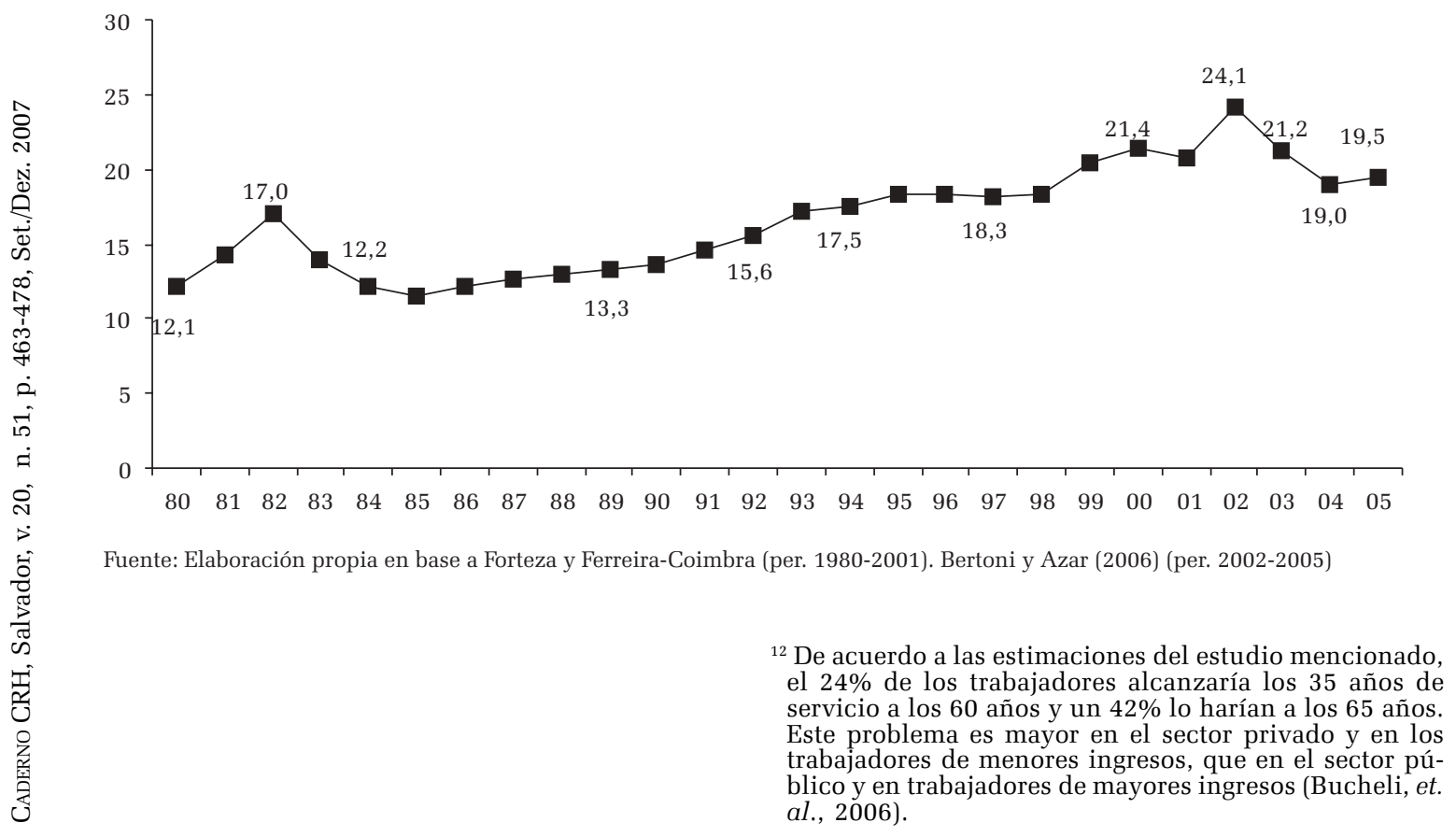


(GPS) en relación al producto bruto interno (PBI) en las últimas décadas. ${ }^{13} \mathrm{Al}$ contrario de lo que se podría imaginar, en la fase reformista que se produjo durante los noventa, el gasto público social presentó una evolución creciente en términos del PBI. Éste se incrementó significativamente en el período 1990-1995, pasando de ubicarse en un $13,7 \%$ en el primer año a $18,3 \%$ en el último, principalmente como consecuencia del aumento del gasto en Seguridad Social (producto del mecanismo de reajuste de las jubilaciones y pensiones, así como por la necesidad de financiar los costos de transición hacia el nuevo sistema provisional). (Flood; Grau; Melgar, 2005).

En el contexto del gobierno frenteamplista se advierte una leve expansión de la relación GPSPBI, situándose en 19,5\% en el año 2005, tendencia que parece consolidarse a partir de la Ley Presupuestal 2005-2009 (Bertoni; Azar, 2006) y del proyecto de Ley de Rendición de Cuentas 2006. Específicamente, dicho proyecto sugiere el afianzamiento de la tendencia a dotar de una mayor prioridad macroeconómica al GPS. ${ }^{14}$ En particular, cabe destacar las partidas presupuestales destinadas a financiar nuevas iniciativas - Plan de Equidad -, las dirigidas a la Educación, así como a impulsar reformas en sectores tradicionalmente bloqueados como el de Salud.
Al observar la distribución sectorial del GPS (Gráfico 5) se advierte que éste se concentra fuertemente en Seguridad Social (14,4\% respecto del PBI en el promedio del período 2002-2005), seguido por Educación (en torno al 3\% del PBI) y Salud (1,9\% del PBI en el promedio de período) (Bertoni; Azar, 2006).

Resulta probable que el conjunto de medidas sociales impulsadas por el actual gobierno no modifiquen sustancialmente la estructura sectorial de asignación del gasto, aunque se registren algunos incrementos en sectores específicos, como, por ejemplo, educación.

\section{EL SIGNIFICADO POLÍTICO DE LAS INICIATI- VAS SOCIALES DE LA IZQUIERDA}

Resulta evidente que la izquierda operó fundamentalmente en dos campos sociales estratégi-

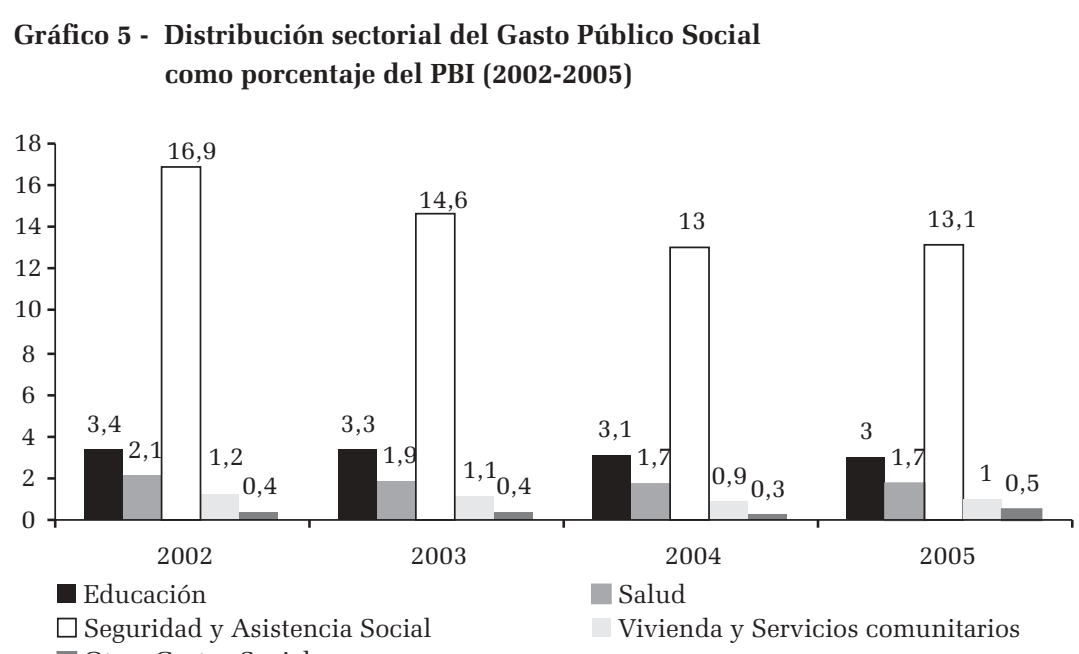

Otros Gastos Sociales

Fuente: Elaboración propia sobre la base de Bertoni y Azar (2006). cos, el del trabajo y el de la pobreza, conectados supuestamente por una iniciativa tributaria, que, entre otras cosas, tiende a favorecer la redistribución social.

Es posible considerar que el inicio del gobierno - con el lanzamiento simultáneo de dos propuestas sociales, los Consejos deSalarios yel Plan deEmergencia - pareciócorresponderse con un tiempopolítico parti- 
cular, caracterizado por fuertes expectativas ciudadanas sobre los cambios de orientación que introduciría la nueva Administración en materia social.

Cabe suponer, además, que la puesta en práctica de esas dos iniciativas definió, en la práctica, un "timing" escalonado de implementación de proyectos sociales, centrados, en una primera instancia, en la mejora salarial y en la atención a las situaciones de pobreza, para pasar posteriormente a la puesta en práctica de modificaciones sustantivas en el sistema de tributación nacional. Ese "timing" reformista condiciona las posibilidades efectivas de instrumentar cabalmente nuevas propuestas sociales - como la ya anunciada de salud -, no solo por el período que resta de mandato, sino por la "pesada" administración de los costos políticos acumulados que emergen de los ajustes y cambios en el esquema de prestaciones sociales. En este sentido, los fuertes vetos políticos podrían traducirse, en el futuro inmediato, en "castigos" electorales que le impedirían que la izquierda se posicionara como una candidata favorita para un segundo gobierno. (Pierson, 2001).Por esta razón, cabe esperar que de la proyectada reforma del sector salud sólo se lleve a cabo en una porción relativamente pequeña.

Resulta evidente que una serie de revisiones de importantes políticas sociales no formaron parte de la agenda de cambio del gobierno, como es el sistema educativo y el de seguridad social. Seguramente esta conducta se explique por diversas razones, entre las que importa destacar, para el caso de la educación, el fortalecimiento de los gremios docentes a partir de la modalidad "dirigista" de implementación que tuvo la reforma educativa. Ese fortalecimiento gremial se traduce sistemáticamente en altas cuotas de conflictividad y desacuerdo político frente a cualquier modificación que se pretenda introducir en el sector.

El mantenimiento del sistema de seguridad social parece corresponderse con otras causas, las que se detallaron anteriormente, no obstante podrían resumirse en la operativa del "pequeño" legado históricoquesegeneróen estos diezaños defuncionamiento del nuevo régimen jubilatorio y, en consecuencia, las resistencias a incorporar más transformaciones.
Ahora bien, interesa descifrar el sentido político de las medidas sociales promovidas por la Administración de izquierda, así como los factores explicativos que incidieron en la definición de esa estrategia de acción.

En este sentido, es posible considerar que las políticas sociales impulsadas por la izquierda uruguaya supusieron a grandes rasgos una "recalibración" del sistema de bienestar que disponía el país, ya sea restaurando los clásicos mecanismos de protección-Consejos de Salarios $-y(0)$ innovando en el plano de la asistencia social - Planes de Emergencia y Equidad (Pierson, 2001; Midaglia; Antía, 2007). El mencionado ajuste del sistema resposicionó al Estado en tanto un agente importante en la provisión de bienes sociales.

No hay lugar a dudas que las principales variables que jugaron un papel de relevancia en la construcción y puesta en práctica de ese conjunto de iniciativas por parte del gobierno de izquierda fueron: sus bases ideológicas y programáticas a favor del trabajo, de la eliminación o limitación de la exclusión social y de la redistribución social; la preeminencia de una cultura política relativamente estatista aún con cierta vigencia en el país (Filguiera et al., 1989); la ausencia de un fuerte sector político de orientación liberal, y la facilidad que le otorgó disponer de mayorías parlamentarias para tramitar políticas públicas de cierta conflictividad. (Aust; Arriba, 2001).

\section{CONSIDERACIONES FINALES}

Es correcto afirmar que la izquierda intervino en materia social, esencialmente en el plano de laboral y el de la pobreza, reutilizando algunos mecanismos tradicionales, así como impulsando planes sociales de amplia cobertura e iniciativas tributarias que no figuraban en la historia del bienestar nacional. De esa forma, la actual Administración tendió a diferenciarse de las anteriores, redefiniendo el papel de la esfera pública en el campo social.

En ese sentido, la direccionalidad que 
asumieron los cambios planteados por este gobierno tendió a enfatizar el rol del Estado en tanto un agente relevante de protección social, ya sea regulando la fijación de las pautas salariales, responsabilizándose por la atención de las situaciones de pobreza y vulnerabilidad, así como también determinando las nuevas pautas distributivas. Es posible afirmar, que la izquierda uruguaya promovió, en torno al sistema de políticas sociales en general, un estatismo moderado, pese a que algunos de los servicios semiprivatizados se mantuvieron sin mayores modificaciones - seguridad social.

Por último, interesa señalar que la puesta en práctica de la reforma tributaria en términos de una política pública de envergadura que tiende a incidir en la matriz de bienestar vigente, se presenta como una apuesta arriesgada del gobierno. Es evidente que la modificación sustantiva de las pautas de tributación produce cierto descontento social, en particular entre los sectores medios y mediosaltos afectados por dichos cambios. Esos segmentos sociales son los que disponen de mecanismos de presión y de formación de opinión pública que pueden deslegitimar la posición que asumió el gobierno en esta materia. La generación de una situación de esa naturaleza podría producir una pérdida de apoyo ciudadano al gobierno y por ende de futuros "castigos" electorales.

En el momento actual se registra, en torno a esta medida, un conjunto de planteos críticos de parte de diversos actores colectivos, no obstante la Administración de Izquierda parece estar decidida a correr s riesgos y, en algún sentido, a modificar las preferencias de la ciudadanía, de igual modo que se hizo en el pasado reciente, cuando sólo era gobierno de la ciudad de Montevideo.

Resulta correcto afirmar que el gobierno posee niveles significativos de fortaleza para sostener sus posiciones y sobre todo parece estar convencido que algunos cambios son esenciales para establecer pautas mínimas de justicia social.

(Recebido para publicação em setembro de 2007)

(Aceito em dezembro de 2007)

\section{REFERÊNCIAS}

AMARANTE, Verónica; VIGORITO, Andrea, Evolución de la pobreza en el Uruguay 2001-2006. Montevideo: Instituto Nacional de Estadística. Programa de las Naciones Unidas para el Desarrollo/UNFPA, 2007.

ARIM, Rodrigo; RUBIO, Mónica; VIGORITO, Andrea. Pobreza, red de protección social y situación de la infancia en Uruguay. Montevideo: BID, 2005. (Serie de Estudios Económicos y Sociales).

ANEP. Gerencia de Investigación y Evaluación. Los niveles de desempeño al inicio de la Educación Primaria. Estudio de las competencias lingüísticas y matemáticas. Evaluación Nacional de Aprendizajes en el primer nivel de la escolaridad. Primer informe. Montevideo, 2002.

AUST, Andreas; ARRIBA, Ana. Policy reforms and discourses in social assistance in the 1990s: towards 'activation'? Madrid: CSIC. Working Paper 04-11, 2004.

BERTONI, Reto; AZAR, Paola. Identificación y análisis del gasto público social en Uruguay 2002-2005. Montevideo: Ministerio de Desarrollo Social/Universidad de la República, 2006

BUCHELI, Marisa et al. El acceso a la jubilación o pensión en Uruguay: ¿Cuántos y quiénes lo lograrían? Documento n.01/06, Montevideo, Departamento de Economía, Facultad de Ciencias Sociales/UdelaR, 2006

BUSQUETS, José Miguel; SETARO, Marcelo. Preparando las ideas y los actores para una futura reforma. Informe de Coyuntura 2007, Montevideo, Instituto de Ciencia Política, Facultad de Ciencias Sociales, Universidad de la República. Ediciones de Banda Oriental, 2007.

CASTELLANO, Ernesto Uruguay: un caso de bienestar de partidos. Revista Uruguaya de Ciencia Política, Montevideo, Ed. ICP-FCS, n.9, 1996.

CASTIGLIONI, Rossana. The politics of social policy change in Chile and Uruguay. Retrenchment versus maintenance, 1973-1998. New York: Routledge, 2005.

CEPAL. La protección social de cara al futuro: acceso, financiamiento y solidaridad. Trigésimo primer período de sesiones de la CEPAL. Montevideo: 2006.

Panorama social de América Latina 2005. División de Desarrollo Social y la División de Estadística y Proyecciones Económicas de la CEPAL. Santiago de Chile: 2006 .

DE ARMAS, Gustavo. De la sociedad hiperintegrada al país fragmentado. Crónica del último tramo de un largo recorrido. En: CAETANO, G. (Ed.) 20 años de democracia: Uruguay 1985-2005: miradas múltiples. Montevideo: Ediciones Santillana, 2005

DEL PINO, Eloisa; COLINO, César. ¿Cómo y por qué se reforman los Estados de bienestar? Avances y retos teóricos y metodológicos en la agenda de investigación actual. En: ; $\quad$ (Coords.) Zona abierta 114/115. La reforma del $\overline{\text { Estado }}$ de bienestar. Madrid: 2006.

FERNÁNDEZ, José Enrique; MOREIRA, Constanza; MITJAVILA, Myriam. Evaluación de la reforma de salud en Uruguay: equidad y redefinición del Welfare State. Montevideo: CIESU-Centro de Informaciones y Estudios del Uruguay, 1999

FERREIRA-COIMBRA, Natalia; FORTEZA, Alvaro. Protección social en Uruguay: financiamiento, cobertura y desempeño 1990-2002. Santiago de Chile: Oficina Internacional del Trabajo, 2004.

FILGUEIRA, Carlos H. et al. De la transición a la consolidación democrática: imágenes y cultura política en el Uruguay. Serie Informes, Montevideo, CIESU, n.38, 1989. 
FILGUEIRA, Fernando. El nuevo modelo de prestaciones sociales en América Latina: residualismo, eficiencia y ciudadanía estratificada. En: BRIAN, Roberts (Ed.) Ciudadanía y Política Sociales. San José de Costa Rica: FLACSO/SSRC, 1998.

FLOOD, Cristina; GRAU, Carlos; MELGAR, Alicia. Análisis del gasto público social. Washington: BID, 2005. (Serie de estudios económicos y sociales).

INSTITUTO DE ECONOMÍA. Consideraciones sobre los cambios en la línea de pobreza del INE (2002). Montevideo: Facultad de Ciencias Económicas y de Administración. Facultad de Ciencias Sociales. Universidad de la República, 2003. (Serie documentos de trabajo DT 06/03).

INE. Evolución de la pobreza por el método del ingreso. Uruguay 1986-2001. Montevideo: 2002.

KATZMAN, Ruben; RODRÍGUEZ, Federico. Encuesta Nacional de Hogares Ampliada. Situación de la Educación en Uruguay. Montevideo: UNFPA, INE, PNUD. 2007.

LANZARO, Jorge La reforma educativa en Uruguay (19952000): virtudes y problemas de una iniciativa heterodoxa. Santiago de Chile: CEPAL, 2004. (Serie de políticas sociales)

MANCEBO, M. Ester; BENTANCUR, Nicolás. Políticas educativas 2006. Informe de Coyuntura n.7, Montevideo, Instituto de Ciencia Política, Facultad de Ciencias Sociales, Universidad de la República, Ediciones de Banda Oriental. 2007. (En prensa).

MIDAGLIA, Carmen. Plan de equidad. Informe de Coyuntura n.7, Montevideo, Instituto de Ciencia Política, Facultad de Ciencias Sociales, Universidad de la República. Ediciones de Banda Oriental, 2007 (En prensa)

El plan de emergencia. Informe de Covuntura n.6. ¿Y ahora? El primer ciclo del gobierno de izquierda en Uruguay, Montevideo, Instituto de Ciencia Política, Facultad de Ciencias Sociales, Universidad de la República. Ediciones de Banda Oriental, 2006.

Alternativas de protección a la infancia carenciada. $\overline{L a}$ peculiar convivencia de lo público y lo privado en el Uruguay. Buenos Aires: Ed. CLACSO/Assdi, 2000.

; ANTÍA, Florencia. La Izquierda en el gobierno: ¿cambio o continuidad en las políticas de bienestar social?. Revista Uruguaya de Ciencia Política $n^{\circ} 16$. Montevideo, Instituto de Ciencia Política, Ediciones Cauce, 2007.

; CASTILLO, Marcelo. Reforma social y sistema de protección: argumentos para la direccionalidad política de las intervenciones públicas. Guadalajara: Universidad de Guadalajara, 2007 (En prensa).

(Coord.); ANTÍA, Florencia; CASTILLO, Marcelo Las tercerizaciones de las prestaciones sociales: un debate político inconcluso. Montevideo: Informe de Consultoría, Oficina de Planeamiento y Presupuesto, 2006.

MINISTERIO DE DESARROLLO SOCIAL. Universidad de la República. Repertorio de políticas sociales, Montevideo: 2007. (Informe de la $1^{\mathrm{a}}$ etapa).

\section{MINISTERIO DE ECONOMÍA Y FINANZAS. Proyecto de} Ley de Rendición de Cuentas. Montevideo: 2007. (Informe Económico)

. Los beneficios del nuevo sistema tributario, 2007. Disponible en: www.uruguayavanza.gub.uy.
Proyecto de Ley de Reforma del Sistema Tributario. Montevideo: 2006. (Exposición de motivos).

MINISTERIO DE TRABAJO Y SEGURIDAD SOCIAL. Memoria Anual del MTSS. Montevideo: 2006.

MINISTERIO DE SALUD PÚBLICA. Proyecto de Ley de Creación del Sistema Nacional Integrado de Salud, Montevideo: [s.d.]

Proyecto de Ley Administración de los Servicios de Salud del Estado. Creación como servicio descentralizado. Montevideo: [s.d.].

Proyecto de Ley Creación del Fondo Nacional de Salud. Montevideo: [s.d]

MOREIRA, Constanza. El voto moderno y el voto clasista revisado. Explicando el desempeño electoral de la izquierda en las elecciones de 2004 en Uruguay. En: Las claves del cambio. Montevideo: ICP-EBO, 2005.

: SETARO, Marcelo. Alternativas de reforma del sistema de salud en Uruguay. En: MANCEBO, M.E; NARBONDO, P.; RAMOS, C. (Comps), Uruguay: la reforma del estado y las políticas públicas en la democracia restaurada (1985-2000). Montevideo: Ed. Banda Oriental/ICP, 2000. (Colección política viva).

MOREIRA, Fernando. Uruguay: ¿Un Estado socialdemócrata? 2003. Tesis (Licenciatura en Ciencia Política) - ICP/FCS/Udelar, Montevideo. (Mimeo.).

NOTARO, Jorge. Los Consejos de salarios en el Uruguay 2005-2006. Serie Documentos de Trabajo DT 04/07. Montevideo, Instituto de Economía, Facultad de Ciencias Económicas y de Administración, Universidad de la República, 2007.

OLESKER, Daniel. Entrevista concedida a Radio Espectador. Montevideo, 7 jun. 2007. Disponible en: www.espectador.com

PERAZZO, Ivone; RODRÍGUEZ, Sandra. Anexo - impactos de la reforma tributaria sobre los ingresos de los hogares. Montevideo: Instituto de Economía, Facultad de Ciencias Económicas y de Administración/UdelaR, 2006.

PEREIRA, Javier; MONTEIRO, Lucía; GELBER, Dense. Cambios estructurales y nueva configuración de riesgos: desbalances e inequidades en el sistema de salud uruguayo. Revista Prisma: dilemas sociales y alternativas distributivas en Uruguay. Montevideo: Universidad Católica del Uruguay, n.21, 2005.

PIERSON, Paul. Sobrellevando la austeridad permanente. Reestructuración del Estado de bienestar en las democracias desarrolladas. En: DEL PINO, Eloisa; COLINO, César (Coords.) Zona abierta 114/115. La reforma del Estado de bienestar. Madrid: 2001.

SENATORE, Luis. "Las relaciones laborales". Informe de Coyuntura n.7, Montevideo, Instituto de Ciencia Política, Facultad de Ciencias Sociales, Universidad de la Repúbli$\mathrm{ca}$,/Ediciones de Banda Oriental. 2007 (en prensa).

SOLARI, Aldo. El desarrollo social del Uruguay en la postguerra. Montevideo: Ed Alfa, 1967.

TRYLESINSKI, Fanny. Los uruguayos y la salud: situación, percepciones y comportamiento.Montevideo: INE. 2007. (Encuesta Nacional Ampliada de Hogares 2006). 
UMA NOVA SÍNTESE? a agenda social da esquerda uruguaia em dois anos de governo

\section{Carmen Midaglia}

Florencia Antía

$\mathrm{O}$ artigo analisa as principais políticas de bem-estar desenvolvidas pelo governo de esquerda no Uruguai, nos dois primeiros anos de mandato, procurando identificar as que foram priorizados, a that were prioritized, the predominant orientação predominante e o papel que o orientation and the role that the State plays Estado desempenha na provisão da in the provision of the social protection. proteção social. Os resultados preliminares The preliminary results indicate the indicam a configuração de um estadismo configuration of a moderate statism in gemoderado em matéria de políticas públicas neral regarding public policies and, in partiem geral e, em particular, no campo social. cular, in the social field. However, the Entretanto, constata-se a persistência de persistence of a group of strategic social um conjunto de serviços sociais estratégi- services is verified, such as the system of cos, como o sistema de seguridade social, social security, that continue in a que continuam num regime de semiprivatization regime, such as was semiprivatização, instaurado em governos established in previous governments. This anteriores. Essa convivência de opções coexistence of different options, in the dessemelhantes, no campo social, abre um social field, opens a group of political issues conjunto de questões políticas sobre o on the system of emerging social politics. sistema de políticas sociais emergentes.

Palavras-Chave: políticas sociais, bem es- Keywords: social politics, welfare; tar; proteção, vulnerabilidade, pobreza, protection, vulnerability, poverty, public gasto público. expense.
UNE NOUVELLE SYNTHÉSE? L'agenda social de la gauche uruguayenne pendant deux ans de gouvernement

Carmen Midaglia

Florencia Antía

Dans cet article, on analyse les principales politiques du bien-être socia développées par le gouvernement de gauche en Uruguay, au cours de ses deux premières années de mandat, en essayant d'identifie les domaines et les secteurs qui ont été prioritaires, l'orientation qui a prédominé et le rôle de l'Etat en ce qui concerne la protection sociale. Les premiers résultats indiquent un gouvernement modéré en matière de politiques publiques en général et dans le domaine social en particulier. On peut cependant constater la persistance d'un ensemble de services sociaux stratégiques, el le système de sécurité sociale, qui continuent en régime semi privé et qui ont été instaurés par les gouvernements précédents. Ce mélange d'options aussi peu ressemblantes, dans le domaine social, débouche sur un ensemble de ques-tionnements politiques relatifs au système des politiques sociales émergentes.

Mots-clés: politiques sociales, bien-être protection, vulnérabilité, pauvreté, coût social. 